\section{THE FUTURE OF FORESTRY IN CANADA}

I would like to thank the members of the CIF for the honour given me when I was elected a member of the Executive Committee in 1990 .

During the next year, you will have an opportunity to review the wisdom of your choice.

Each member of the Executive brings their strength, weakness and interests to the leadership. All of us are different. When I ran for election I did so on a platform of participating in the evolution of Canadian forestry practices - specifically:

- Integrated Forest Resource Management

- Reduction of Environmental Impacts of Woodlands Operations

- Improvements to Tenure Arrangements

- Stable Funding for Silviculture

- Forest Crop Planning

Forestry in Canada is in a period of drastic and rapid change. Many of these changes are painful. I believe the changes are driven by the following factors:

1. Public concern about the "loss of nature",

2. The ground swell of public support for a better environment.

3. The communications revolution video/TV.

4. The concept of sustainable development.

5. The new understanding and support for biodiversity and wildlife habitat.

6. Consumer power.

7. GATT and trade related environmental measures.

8. Rio' 92 and follow-up procedures.

9. Codes of practice for foresters and forest operations.

If our evolution is being propelled by these forces then the direction of change will be:

1. Public involvement in forest management planning.

2. Integrated forest resource management founded on forest ecology.

3. Woodlands operations planned and carried out with greater concern for aesthetics, impacts on water quality and wildlife habitat.

4. The establishment of standards by which the forest resource stewardship of countries and companies will be judged and, "Greenmarks", awarded for good performance.

These, "Greenmarks", will facilitate entry into the market place.

What steps must we take to move in these new directions?

I suggest the following:

1. Completion and use of forest ecosystem classification systems for all regions of Canada.

2. Woodlands operation plans and the choice of silviculture systems should be based more on forest science and less on tradition.

3. Excellent inventories and good growth and yield data.

4. Consider silviculture trust funds held by a third party

\section{L'AVENIR DE LA FORESTERIE AU CANADA}

J'aimerais remercier les membres de l'IFC pour l'honneur qui m'a été donné d'avoir été élu membre du Comité exécutif en 1990.

Au cours de la prochaine année, vous aurez la possibilité d'analyser le bien fondé de votre choix.

Chacun des membres de l'Exécutif assume son leadership en fonction de ses forces, de ses faiblesses et de ses intérêts. Nous sommes tous différents. Lorsque que j'ai sollicité ce mandat, je le faisais dans le but de participer à l'évolution des pratiques forestières au Canada, et plus spécifiquement au niveau de:

- l'aménagement intégré des ressources forestières

- la réduction des impacts environnementaux des opérations forestières

- l'amélioration des conditions de tenure

- un financement stable pour la sylviculture

- la planification de la récolte forestière

La foresterie au Canada vit une période de changements drastiques et rapides. Plusieurs de ces changements se font dans la douleur. Je crois que ces changements découlent des facteurs suivant:

1. La préoccupation du public relativement à "la destruction de la nature"

2. L'expression profonde de l'appui du public pour un meilleur environnement.

3. La révolutions des communications - video/télévision

4. Le concept de développement durable.

5. La nouvelle compréhension et le support de la biodiversité et des habitats fauniques.

6. Le pouvoir des consommateurs.

7. Le GATT et les mesures commerciales reliées à l'environnement.

8. Rio'92 et les procédures de suivi.

9. Les normes d'exercices pour les forestiers et les opérations forestières.

En supposant que notre évolution est dirigée par ces forces, alors la direction du changement reposera sur:

1. La participation du public à la planification de l'aménagement forestier.

2. L'aménagement intégré des ressources forestières basé sur l'écologie forestière.

3. Des opérations forestières planifiées et entreprises avec un grand soucis des aspects esthétiques, des impacts sur la qualité de l'eau et des habitats fauniques.

4. La mise en place de normes par lesquelles la gestion des ressources forestières effectuée par les pays et les compagnies sera évaluée et la remise de "Médailles vertes" pour souligner une bonne performance.

Ces "médailles vertes" faciliteront l'accès au marché.

Quelles sont les étapes à franchir pour prendre ces nouvelles directions? Je suggère les suivantes:

1. Le parachèvement et l'utilisation des systèmes de 
for each management area as a silviculture funding mechanism.

5. Examine the effects of road construction costs on forest land management planning and operations. Seek solutions in fiscal policy.

6. We need new tenure systems to provide industry with the incentives to increase wood production and the ability to manage forest land in harmony with nature and public expectations.

The world around us is changing rapidly.

- Global markets

- The global village

- The communications revolution

- Dangerous increases in world population and rates of development, leave many of us alarmed about the future.

In the past, Canadian forests provided:

- Wood for industry

- Environmental benefits

- Areas for recreation

As the economy of the world and the needs of the exploding world population changes, perhaps the forests of Canada will provide:

- Wood to meet the needs of people

- A tourism resource - a growing part of our economy

- Environmental benefits, which some people term "The Lungs of the Earth"'. The world may pay us to keep these lungs healthy.

We as members of the CIF/IFC must use our knowledge of forest science, our human ingenuity and our influence in the forest sector to promote change and remain competitive.

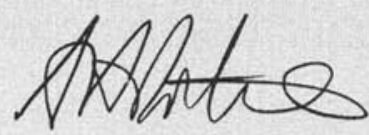

Tony Rotherham, President classification des écosystèmes forestiers pour toutes les régions du Canada.

2. Les plans des opérations forestières et la sélection des systèmes sylvicoles devraient être issus des sciences forestières et moins des habitudes.

3. Il faut obtenir d'excellents inventaires et de bonnes données de croissance et de rendement.

4. Considérer la mise en place de fonds sylvicoles détenus en fiducie par une troisième partie pour chaque unité d'aménagement comme un mécanisme de financement de la sylviculture.

5. Examiner les effets des coûts de construction de route sur la planification de l'aménagement des terres boisées et sur les opérations. Rechercher des solutions au niveau des politiques fiscales.

6. Nous avons besoin de nouveaux systèmes de tenure afin de remettre à l'industrie des incitatifs pour accroître la production de bois et la capacité d'aménager les terres forestières en harmonie avec la nature et les attentes du public.

Le monde qui nous entoure change rapidement.

- Les marchés mondiaux

- Le village global

- La révolution des communications

- L'augmentation inquiétante de la population mondiale et de son taux de développement, laissent plusieurs d'entre nous inquiets face à l'avenir.

Dans le passé, les forêts canadiennes ont procuré:

- Du bois pour l'industrie

- Des bénéfices environnementaux

- Des zones de récréation

A mesure que l'économie mondiale et les besoins d'une population mondiale en explosion se modifieront, les forêts du Canada pourront peut être fournir:

- Du bois pour satisfaire les besoins des gens

- Une ressource touristique - un élément en croissance de notre économie

- Des bénéfices environnementaux, ce que certaines personnes identifient selon l'expression "Les poumons de la Terre". Le monde pourrait nous payer pour maintenir ces poumons en santé.

En tant que membres de l'IFC/CIF nous devons utiliser nos connaissances des sciences forestières, notre ingéniosité humaine et notre influence au sein du secteur forestier afin de promouvoir les changements et demeurer compétitifs.

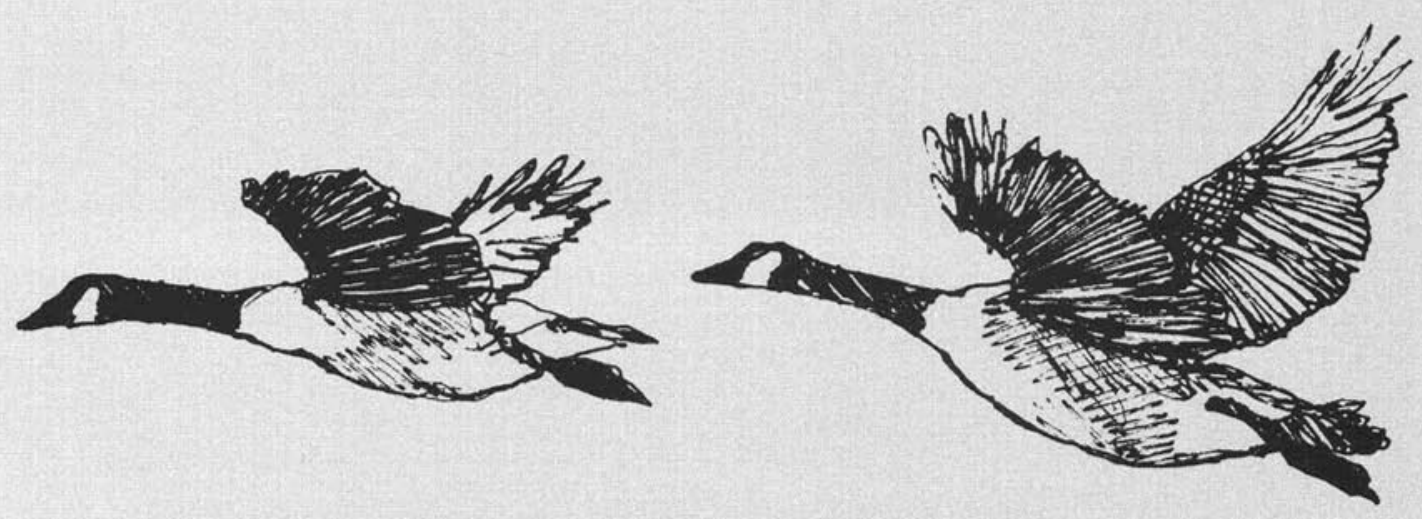




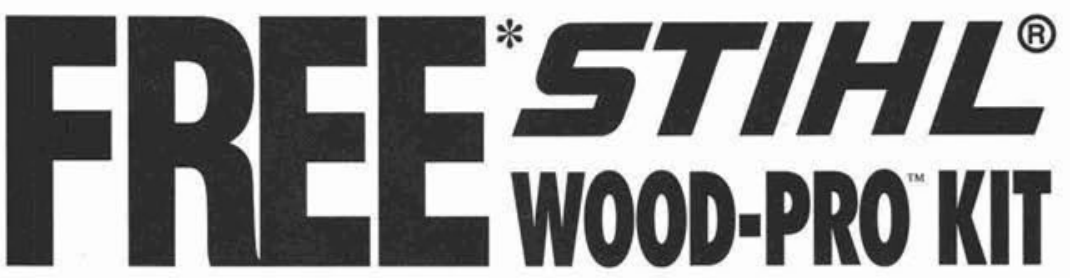

\section{KIT INCLUDES:}

- Oilomatic ${ }^{\circledR}$ Replacement Chain Loop

- 10 Litre Fuel Container with Stop-Flow Spout

- $250 \mathrm{ml}$ Engine Oil Mix

- 1 Litre Chain Oil

- Chain Sharpening File with Handle

- Coleman ${ }^{\circledR}$ Cooler

- STIHL Cap

- 'Sharp Advice' Filing Manual

- Valuable STIHL Coupons
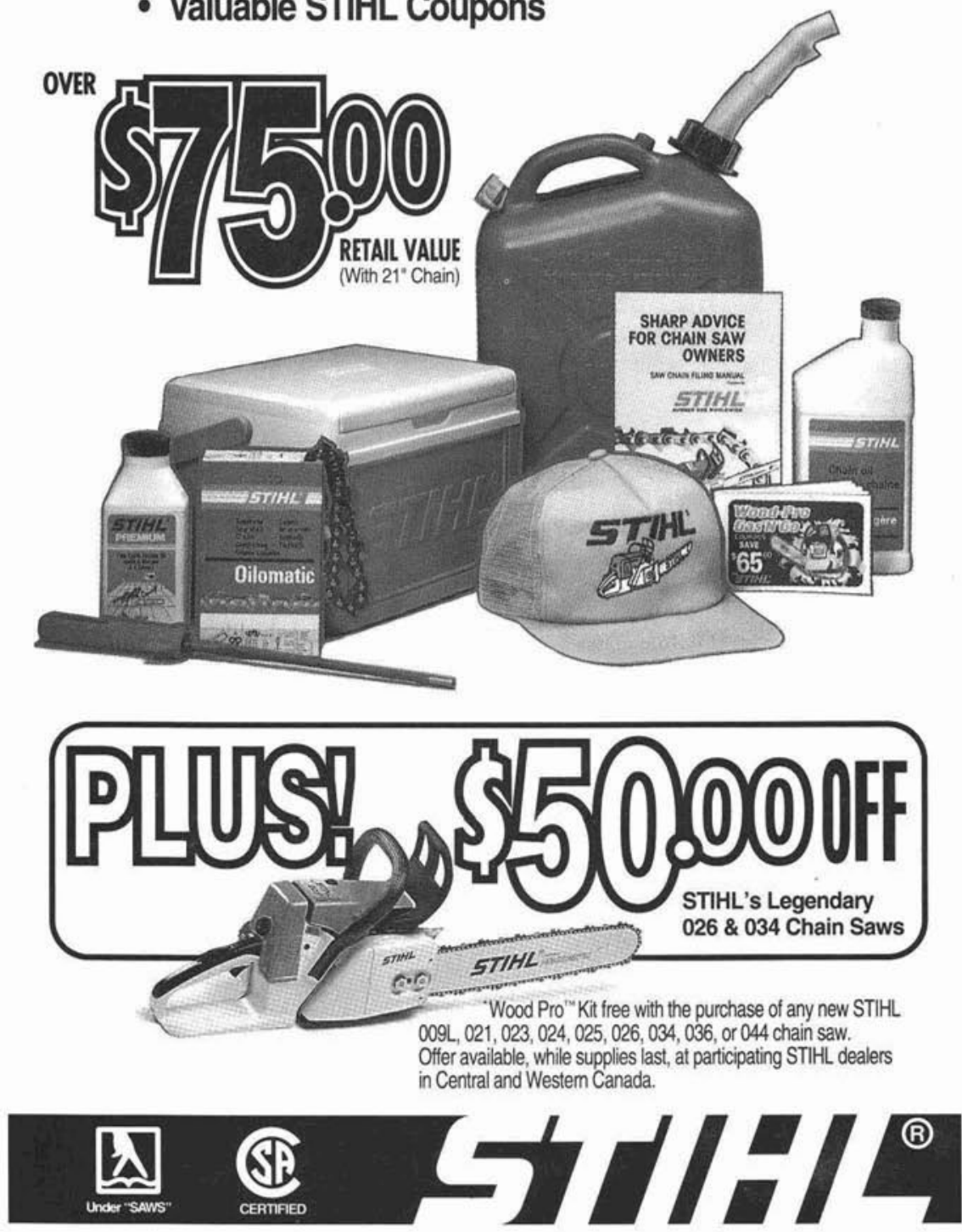

Number One Worldwide 